\title{
Micrometastases in Sentinel Lymph Nodes: Not Getting Lost in Translation
}

\author{
Sandra L. Wong, MD, MS \\ Department of Surgery, University of Michigan, Ann Arbor, MI
}

Advances in technology have led to increasingly minimally invasive procedures and an increased ability to detect very small amounts of disease. With sentinel lymph node (SLN) biopsy, initial concerns about false-negative results are now balanced with concerns of clinical false-positive results, defined as instances when small amounts of disease have no downstream clinical consequences. The evolution in carefully defining what constitutes clinically meaningful disease and necessary treatments is nicely illustrated in breast cancer. SLNs are considered positive if tumor cells meet a size threshold of $0.2 \mathrm{~mm}$ or 200 cells, although disease that is detected by cytokeratin immunohistochemistry alone (without confirmatory hematoxylin and eosin staining) still has somewhat unclear implications. Further, even in the setting of unequivocal disease in SLNs, there are increasing data to support not completing an axillary lymph node dissection (ALND). Most notably, practicechanging results from the American College of Surgeons Oncology Group Z0011 trial effectively demonstrated that ALND did not significantly affect overall or disease-free survival, heralding excellent locoregional control from multimodality treatment of patients with $\mathrm{T} 1 / 2$ invasive breast cancer and positive SLNs who are treated with lumpectomy, radiation therapy (including the low axilla), and adjuvant systemic therapy. ${ }^{1}$

However, an acceptably low threshold below which disease burden does not need to be reported as metastatic disease has not been identified in melanoma. Nevertheless, citing evidence that additional disease is rarely seen when there is a very small burden of disease (most commonly

(C) Society of Surgical Oncology 2012

First Received: 6 December 2011;

Published Online: 10 January 2012

S. L. Wong, MD, MS

e-mail: wongsl@umich.edu defined as $<0.1$-mm deposits), some groups are now routinely not performing completion lymph node dissection (CLND). ${ }^{2,3}$ Further, there have been increasing questions about the benefits of CLND in melanoma because since the great majority of patients with a positive SLN do not have additional nodal disease in the CLND specimen. National data suggest that practice patterns have evolved to commonly deferring CLND in patients with positive SLNs. ${ }^{4}$

In this issue of Annals of Surgical Oncology, Murali and colleagues ${ }^{5}$ report timely and important findings from a high-intensity melanoma SLN sectioning protocol. SLN specimens that were previously reported as having very small $(<0.1-\mathrm{mm})$ deposits of melanoma were reprocessed and extensively examined for additional burden of nodal metastases, as measured by maximum size of the largest deposit, depth of penetration below the lymph node capsule, and intranodal location of disease. With a more extensive sectioning protocol, additional disease was found in a large proportion of SLNs. Clinicopathologic correlation and long-term follow-up demonstrated poorer outcomes in this group of patients, all of whom were originally diagnosed as having a lower SLN tumor burden than would be expected for node-negative patients. These findings dispute the hypothesis that there is a threshold below which very small deposits of melanoma in SLNs may be considered clinically irrelevant.

Just as the practice of not performing ALND will certainly start to expand to breast cancer patient populations beyond those examined in the Z0011 trial, the practice of deferring CLND in melanoma patients may expand because of the temptation to translate data from breast SLN studies to the melanoma population. Appropriate caution must be exercised in this context. Beyond some similarities with the theoretic and technical aspects of the SLN biopsy procedure, it is imperative that SLN results for breast cancer and melanoma be considered separately. Patients must be carefully selected for less invasive treatment options on the basis of 
two main factors: disease biology and effectiveness of the intervention.

\section{DISEASE BIOLOGY}

Murali and colleagues ${ }^{5}$ convincingly demonstrate the importance of accurate staging and the pitfalls of incomplete examination of SLNs. The inability to use clinicopathologic variables to reliably predict which nodepositive patients will go on to have more advanced disease may be related to the inaccurate characterization of the pathologic extent of disease. The European Organisation for Research and Treatment of Cancer Melanoma Group MINITUB registration study ${ }^{3}$ will specifically study those patients with a "minimal" tumor burden, as defined by deposits $<0.1 \mathrm{~mm}$, who do not undergo CLND. However, as the present data show, the underestimation of tumor burden with the detection of additional disease could easily alter decision making and treatment planning.

\section{EFFECTIVENESS OF CLND}

The equivocal effect of ALND on breast cancer survival cannot be translated to melanoma. The finding of additional nodes in an ALND specimen does not necessarily change recommendations for adjuvant therapy in breast cancer because those decisions are influenced by a variety of other clinicopathologic factors. Further, there are extremely low axillary failure rates after various treatments for nodal disease in breast cancer (including ALND, radiation, systemic therapy, and even observation). The effectiveness of multimodality treatments for breast cancer is incontrovertible.

However, understanding that there is an imperfect correlation between staging and prognosis, melanoma staging is directly affected by the number of nodes containing metastatic disease. Despite promising results with novel targeted agents for metastatic melanoma, these new systemic options have no currently defined role in the adjuvant setting, but the prospect of upcoming clinical trials calls for a more thoughtful approach to defining what constitutes a positive SLN. The effect of regional control on improving overall survival and the ability to alter the course of disease with CLND are hotly debated, but the extent of survival benefit with CLND remains unknown. Although the Multicenter Selective Lymphadenectomy Trial II, the randomized controlled trial meant to address this very issue, is still accruing patients, practice patterns may be changing ahead of the evidence base, or possibly with data that should not even be considered circumstantial.

In breast cancer, nodal micrometastases don't register ("less is zero"), and ALND is not always necessary ("less is more"). As the current data point out, there may be a systematic underestimation of the extent of nodal metastases in melanoma, and we cannot disregard even a very small amount of disease. It may be premature to say that "more is more", but at the very least, we should call a spade a spade and treat micrometastatic disease as metastatic disease.

\section{REFERENCES}

1. Giuliano AE, Hunt KK, Ballman KV, et al. Axillary dissection vs no axillary dissection in women with invasive breast cancer and sentinel node metastases: a randomized clinical trial. JAMA. 2011;305:569-75.

2. vanAkkooi AC, deWilt JH, Verhoef $\mathrm{C}$, et al. Clinical relevance of melanoma micrometastases $(<0.1 \mathrm{~mm})$ in sentinel nodes: are these nodes to be considered negative? Ann Oncol. 2006;17:1578-85.

3. European Organisation for Research and Treatment of Cancer Melanoma Group MINITUB Registration Study. http://www. melanomagroup.eu/content/view/101/117/. Accessed Nov 2011.

4. Bilimoria KY, Balch CM, Bentrem DJ, et al. Complete lymph node dissection for sentinel node-positive melanoma: assessment of practice patterns in the United States. Ann Surg Oncol. 2008;15: $1566-76$.

5. Murali R, DeSilva C, McCarthy SW, et al. Sentinel lymph nodes containing very small $(<0.1 \mathrm{~mm})$ deposits of metastatic melanoma cannot be safely regarded as tumor-negative. Ann Surg Oncol. (in press). 